\title{
The effects of vitamin D3 supplementation on insulin resistance and glycaemic control in individuals at high risk of type 2 diabetes: a systematic review and meta-analysis
}

\author{
R. McFadden ${ }^{1}$, A. Darling ${ }^{1}$ and S. Lanham-New ${ }^{1}$ \\ ${ }^{1}$ Nutrition, Food and Exercise Sciences Department, School of Biosciences and Medicine, Faculty of Health and \\ Medical Sciences, University of Surrey, Guildford, UK
}

Vitamin D deficiency may have an important role in the development of Type-2 diabetes ${ }^{(1,2)}$. Low vitamin D concentration has broadranging effects that are both directly (by activation of the vitamin D receptor) and indirectly (by modulation of intracellular calcium concentration) related to impaired pancreatic $\beta$-cell function and insulin resistance ${ }^{(3)}$. The purpose of this systematic review and meta-analysis was to examine the effect of vitamin D supplementation on insulin resistance and glycaemic control in participants with prediabetes.

A systematic search of the available evidence was conducted using the ISI Web of Knowledge and the Cochrane Central Register of Controlled Trials (January 2006 - May 2020). We included randomized intervention trials assessing the effects of vitamin D3 supplementation (with or without calcium), compared to placebo, in adults with prediabetes. Included studies needed to report at least one of the co-primary outcomes of interest: fasting plasma glucose (FPG); 2-hour plasma glucose after an oral glucose tolerance test (OGTT); haemoglobin A1C (HbA1c); insulin resistance (HOMA-IR) and insulin sensitivity and secretion (IG Index). Study results were extracted, and methodological quality was assessed. All procedures performed in studies involving human participants were in accordance with the ethical standards of the institutional and/or national research committee and with the 1964 Helsinki Declaration and its later amendments or comparable ethical standards.

Seventeen studies were included in the systematic review. The meta-analysis was performed on ten studies and showed a modest reduction in FPG (Weighted Mean Difference (WMD) (random) $\left.=-0.09 \mathrm{mmol} / 1(95 \% \mathrm{CI}-0.17,-0.00) \mathrm{P}=0.05 \mathrm{I}^{2}=52 \%\right)$ compared to control after vitamin D3 supplementation. However, there was no effect on any other primary outcomes $(\mathrm{P}>0.05)$. In the studies that included a calcium supplement, there was a statistically significant reduction in HbAlc (WMD (random) $=-0.06 \%(95 \% \mathrm{CI}-0.11$, $-0.00, \mathrm{P}=0.03)$. The mixed effects meta-regression revealed that as serum 25 -hydroxyvitamin $\mathrm{D}$ increased by $10 \mathrm{nmol} / \mathrm{L}, \mathrm{FPG}$ decreased by $0.10 \mathrm{mmol} / \mathrm{L}$ (estimate(SE) for $1 \mathrm{nmol} / 125(\mathrm{OH}) \mathrm{D}$ increase $=-0.01(0.005), \mathrm{P}=0.046, \mathrm{I} 2=33.9 \%, \mathrm{R} 2=42.4 \%$ ).

We report a modest reduction of FPG after vitamin D3 supplementation in subjects with prediabetes, albeit with significant heterogeneity between studies. However, there was no effect on other indices of insulin resistance and glycaemic control. Studies were of relatively short duration and small sample size. Study participants were not always vitamin D deficient. Larger and longer randomized clinical trials, that accurately measure serum 25-hydroxyvitamin D and record patient important outcomes, are now needed to further investigate the role of vitamin $\mathrm{D}$ in the development of Type-2 diabetes, especially in patients with vitamin D deficiency.

\section{References}

1. Pittas AG, Dawson-Hughes B, Sheehan P, Ware JH, Knowler WC, Aroda VR, et al. (2019) N Engl J Med; 381(6):520-530.

2. Kayaniyil S, Vieth R, Retnakaran R, Knight JA, Qi Y, Gerstein HC, et al. (2010) Diabetes Care; 33(6):1379-1381.

3. Norman AW, Frankel JB, Heldt AM, Grodsky GM. (1980) Science; 209(4458):823-825. 